\title{
Previsão espacial dos teores de elementos-traço em depósitos de veículos com base em modelagem geoestatística
}

Metais pesados podem ocorrem naturalmente nos solos provenientes do seu material de origem. Infelizmente, o número de áreas contaminadas por processos antropogênicos tem crescido e gerado graves problemas socioambientais, provocando riscos ecológicos e para a saúde humana. O objetivo principal deste estudo foi investigar a concentração de elementos-traços presentes em amostras de solo através de uma análise espacial utilizando técnicas geoestatísticas. Para a realização desta modelagem foram analisadas amostras de solo coletadas em duas áreas sem impermeabilização de depósito de veículos apreendidos pela Polícia Rodoviária Federal localizados às margens da rodovia BR-476 (Rodovia do Xisto) na região de Araucária e BR-116 na região de Colombo, ambas no estado do Paraná, região metropolitana de Curitiba. As amostras foram preparadas conforme metodologia de digestão ácida e a leitura dos metais Pb, Zn, $\mathrm{Cr}$, Cu e Ni realizada por Espectrofotometria de Emissão Óptica por Plasma Indutivamente Acoplado. Para a realização das análises estatísticas descritiva e espacial, análise variográfica e geração dos mapas das estimativas, foi utilizado o software $\mathrm{R}$ com o pacote geoR. Após uma análise descritiva dos cenários para verificar a existência de dependência espacial, construiu-se os variogramas experimentais que foram ajustados pelos modelos esférico, exponencial e gaussiano. Os mapas gerados utilizando a krigagem ordinária mostram claramente a variação espacial dos elementos destacando os locais com maiores valores de concentração. Além disso, permitem uma leitura de regiões com valores altos do contaminante, sendo muito úteis em estudos, uma vez que auxiliam na tomada de decisão no que tange saúde e ambiente.

\section{Spatial prediction of trace elements in vehicle deposits based on geostatistical modeling}

\begin{abstract}
Heavy metals can occur naturally in soils, from their source material. Unfortunately, the number of areas contaminated by anthropogenic processes has grown and has caused serious socio-environmental problems, causing ecological and human health risks. The main objective of this study was to investigate the concentration of elements present in soil samples through spatial analysis using geostatistical techniques. To carry out this modeling, soil samples collected in two areas without waterproofing vehicle deposits seized by the Federal Highway Police, on the margins of the BR-476 highway (Rodovia do Xisto) in the region of Araucária and BR-116 in the region of Colombo, were analyzed, both in the state of Paraná, metropolitan area of Curitiba. The samples were prepared according to the acid digestion and metal reading methodology for $\mathrm{Pb}, \mathrm{Zn}, \mathrm{Cr}, \mathrm{Cu}$ and Ni performed by Inductively Coupled Plasma Optical Emission Spectrophotometry. For the realization of statistical and spatial statistics, variographic analysis and the generation of scene maps, the software $\mathrm{R}$ alongside the geo $\mathrm{R}$ package was used. After a descriptive analysis of the scenarios to verify the presence of spatial dependence, the experimental variograms that were adjusted by the spherical, exponential and Gaussian models are constructed. The maps generated using ordinary encryption clearly show the spatial variation of the highlighted elements in places with the highest concentration values. In addition, it is possible to create a mapping of regions with high levels of contaminants, being very useful in studies, since it helps in decision making with regard to health and the environment.
\end{abstract}

Keywords: Environmental Impact Assessment; Monitoring; Soils; Spatial Inference; Trace Elements.

Topic: Engenharia Ambiental

Reviewed anonymously in the process of blind peer

Cleyton Nascimento Makara (iD)

Universidade de São Paulo, Brasil

http://lattes.cnpq.br/7241015092201765

http://orcid.org/0000-0002-8034-2582

cleytonmakara@gmail.com

Gleiceane Libero (iD

Universidade Tecnológica Federal do Paraná, Brasil

http://lattes.cnpq.br/6001936320263973

http://orcid.org/0000-0001-7732-9144

glelibero@gmail.com

Larissa Kummer (iD)

Universidade Tecnológica Federal do Paraná, Brasil

http://lattes.cnpq.br/7892203021390255

http://orcid.org/0000-0002-8282-2045

lkummer@utfpr.edu.br

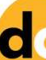

DOI: 10.6008/CBPC2179-6858.2020.005.0030
Received: 05/08/2020

Approved: 26/09/2020

Maurici Luzia Charnevski Del Monego (id

Universidade Tecnológica Federal do Paraná, Brasil

http://lattes.cnpq.br/1410424302920222

http://orcid.org/0000-0002-9093-3802

maurici@utfpr.edu.br

\section{Referencing this:}

MAKARA, C. N.; LIBERO, G.; KUMMER, L.; MONEGO, M. L. C.. Previsão espacial dos teores de elementos-traço em depósitos de veículos com base em modelagem geoestatística. Revista Ibero Americana de Ciências Ambientais, v.11, n.5, p.317-330, 2020. DOI: http://doi.org/10.6008/CBPC2179-6858.2020.005.0029 


\section{INTRODUÇÃO}

A metodologia geoestatística fornece um conjunto de ferramentas que nos permitir investigar a aleatoriedade de um conjunto de dados, os quais apresentam, porém, uma possível estruturação espacial, que pode ser quantificada através de uma função de correlação espacial (YAMAMOTO et al., 2013). As abordagens que têm sido utilizadas para a análise de dados ambientais, como a concentração de elementostraço presentes em solos, não têm sido verdadeiramente esclarecedoras dos reais impactos sobre o ambiente (MONEGO et al., 2010).

O solo é um recurso natural fundamental e muito complexo e um dos componentes de grande importância no ecossistema, pois é a base para a sustentação dos seres humanos, além de ser um suporte ao desenvolvimento de alimentos de origem vegetal e animal, servindo ainda como uma barreira natural à contaminação de águas subterrâneas (PEREIRA et al., 2015).

Os elementos-traço podem ocorrer naturalmente no solo, devido ao material de origem geológico. Entretanto, é cada vez mais frequente a descoberta de áreas contaminadas devido ao uso inadequado do solo, como atividades industriais, uso de fertilizantes químicos, depósito de materiais e resíduos (SOUZA et al., 2018; MILHOME et al., 2018). A preocupação ocorre pelo fato dos metais não se decomporem, podendo acumular-se no solo, na cadeia trófica ou se deslocarem até as águas subterrâneas. Desta forma, tais elementos podem resultar em riscos ecológicos e para a saúde humana (FERNÁNDES, 2017; MILHOME et al., 2018).

A metodologia geoestatística vem sendo aplicada em pesquisas das mais diversas áreas: na modelação da distribuição de elementos-traço ( $\mathrm{As}, \mathrm{Cd}, \mathrm{Cr}, \mathrm{Cu}, \mathrm{Hg}, \mathrm{Ni}, \mathrm{Pb}$ e $\mathrm{Zn}$ ) (LADO et al., 2008); na interpolação de mapas para fins de agricultura de precisão em área de cana-de-açúcar (SOUZA et al., 2014); na avaliação da resistência mecânica do solo à penetração das raízes e do teor de água no solo (BATISTA et al., 2019), entre outros.

A geoestatística pode inclusive ajudar nos processos de remediação destes solos contaminados, uma vez que pode oferecer subsídios para a decisão da melhor técnica e local a ser aplicado, já que traz os dados mapeados (LIN et al., 2002). Estes mapas se tornam ferramentas promissoras para auxiliar a tomada de decisão quanto a questões de políticas públicas relacionadas à saúde e ao planejamento ambiental dos locais contaminados (LOURENÇO et al., 2005).

Neste trabalho foi aplicada a metodologia geostatística para análise de alguns elementos- traço em amostras de solos coletados no pátio de dois postos da Polícia Rodoviária Federal (PRF), nos municípios de Araucária (AR) e Colombo (CL), Paraná.

\section{MATERIAIS E MÉTODOS}

No presente trabalho apresenta-se uma análise geoestatística de um conjunto de dados que foram cedidos pelo Laboratório de Análise de Solos (LASOL) da Universidade Tecnológica Federal do Paraná (UTFPR) câmpus Curitiba. Os dados são referentes a amostras de solo (fração $<2 \mathrm{~mm}$ ) coletadas em duas áreas sem 
impermeabilização de depósito de veículos abandonados e/ou apreendidos da Polícia Rodoviária Federal (PRF) localizados às margens da rodovia BR-476 (Rodovia do Xisto) na região de Araucária (AR) e BR-116 na região de Colombo (CL), ambas no estado do Paraná, região metropolitana da grande Curitiba (Figura 1).

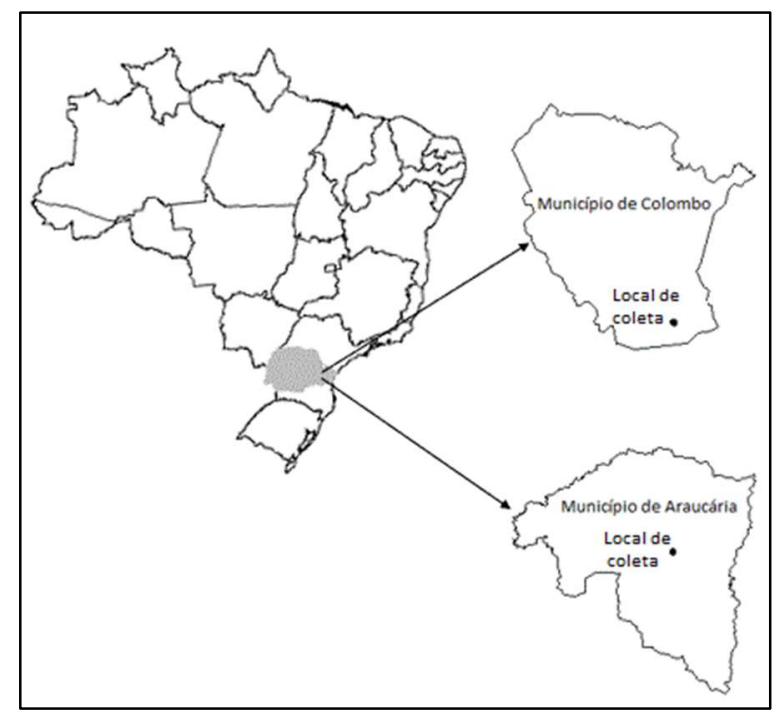

Figura 1: Localização dos pontos de amostragem.

Nas amostras de solo foram analisados os elementos-traço: Cromo $(\mathrm{Cr})$, Cobre $(\mathrm{Cu})$, Níquel $(\mathrm{Ni})$, Chumbo $(\mathrm{Pb})$ e Zinco (Zn), quantificados após análise laboratorial conforme metodologia de digestão ácida e leitura realizada por Espectrofotometria de Emissão Óptica por Plasma Indutivamente Acoplado. O teor indicado para todos os metais analisados está em mg. $\mathrm{kg}^{-1}$ de amostra de solo analisada. Em posse dos conjuntos de dados foram realizadas as análises geoestatísticas com o objetivo de mapear a distribuição dos poluentes nos dois cenários: Cenário 1 - Araucária e Cenário 2 - Colombo. No cenário 1, apresentado na Figura 2, tem-se o município de Araucária.

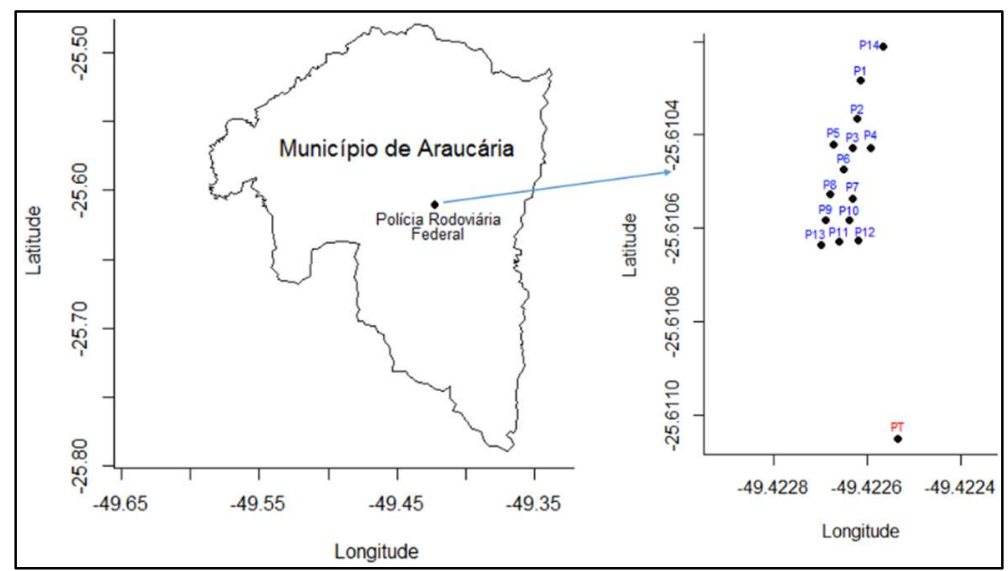

Figura 2: Representação dos pontos de coleta georreferenciados no município de Araucária - Cenário 1.

À direita as localizações devidamente georreferenciados, utilizando coordenadas espaciais (UTM) das 15 amostras de solo superficial coletados. $O$ ponto $P_{\mathrm{T}}$ (em vermelho) no canto inferior da figura à direita, representa um ponto de prova e não participou do cálculo das estimativas para a geração dos mapas da distribuição dos poluentes. Dessa forma, no cenário 1 para a análise geoestatística foram analisados os 14 pontos agrupados no canto superior direito da figura. 
No cenário 2, apresentado na Figura 3, temos o município de Colombo. O ponto preto representa a localização (25o 22' 31,24" S e 49 09’ 5,03” W) do Departamento da Polícia Rodoviária Federal do Paraná (DPRF) às margens da rodovia BR-116 (Rodovia Régis Bittencourt). À direita, as localizações devidamente georreferenciados, utilizando coordenadas espaciais (UTM) das 20 amostras de solo superficial coletados. 0 ponto $\mathbf{P}_{\mathrm{T}}$ (em vermelho) no canto inferior da figura à direita, representa um ponto de prova e não participou do cálculo das estimativas para a geração dos mapas da distribuição dos poluentes. Para a análise geoestatística do cenário 2 foram analisados os 19 pontos distribuídos na área, conforme demostrado à direita da Figura 3.

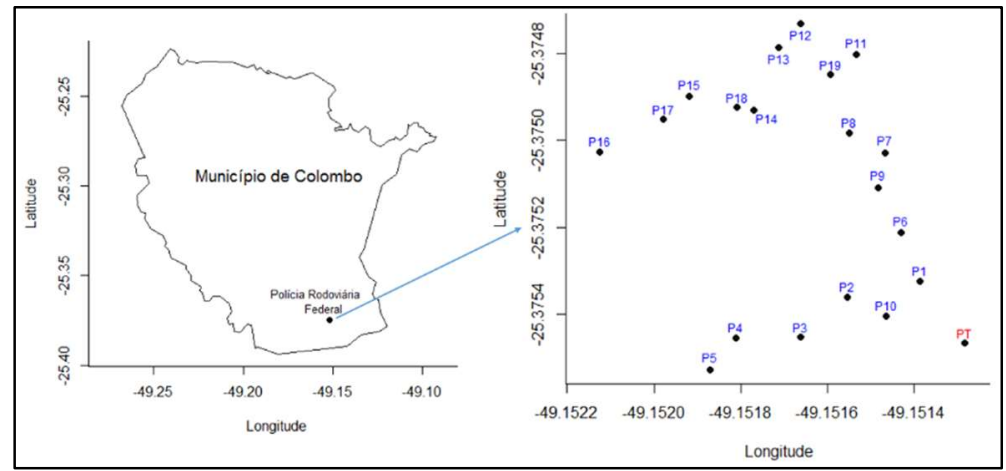

Figura 3: Representação dos pontos de coleta georreferenciados no município de Colombo - Cenário 2.

Para as análises estatística e geoestatística utilizou-se o software R e o package GeoR (R DEVELOPMENT CORE TEAM, 2008). Para os cenários foi realizada, separadamente: a) uma análise estatística descritiva e espacial para obter uma caracterização geral do comportamento da dispersão dos poluentes; b) a construção do semivariograma experimental para a quantificação da dependência espacial observada; c) a modelagem dos semivariogramas experimentais e ajustes a uns dos modelos teóricos: esférico, exponencial ou gaussiano; d) utilizada a técnica de validação cruzada para seleção do modelo teórico que melhor se ajustasse ao conjunto de dados; e) a interpolação através da Krigagem ordinária para gerar os mapas das estimativas.

\section{Análise Estatística Descritiva e Espacial}

Através da estatística descritiva e espacial é possível verificar o comportamento dos dados disponíveis permitindo um diagnóstico do comportamento dos dados, a possível presença de valores anômalos, a existência de assimetrias na distribuição, entre outros. Ainda na mesma etapa faz-se uma análise da existência da dependência espacial das grandezas em estudo. Uma vez detectava a existência de dependência espacial entre os dados, a etapa seguinte é quantificar essa dependência espacial através do semivariograma (CLARK et al., 2000; SOARES, 2000; PEDROSA, 2016; BUSSAB et al., 2017).

\section{Construção do Semivariograma Experimental}

Para modelar o fenômeno espacial através da metodologia geoestatística utiliza-se o semivariograma que permite a quantificação da dependência espacial. De acordo com Soares (2000), na construção do 
semivariograma experimental deve-se levar em conta não só as distâncias entre as localizações e as várias direções. É fato que o fenômeno muitas vezes pode apresentar um comportamento semelhante em todas as direções, e quando isso é observado, o fenômeno é denominado de isotrópico. Mas em certas situações o comportamento da amostragem pode seguir uma determinada direção específica, sendo que neste caso o fenômeno é dito anisotrópico.

A dependência espacial entre as medidas da grandeza em estudo é quantificada através da semivariância de acordo com a equação 1:

$$
\gamma(h)=\frac{1}{2 N(h)} \sum_{i=1}^{N(h)}\left[Z\left(x_{i}\right)-Z\left(x_{i}+h\right)\right]^{2} \text { Equação (1) }
$$

Onde, $Z\left(x_{i}\right)-Z\left(x_{i}+h\right)$ é o valor da diferença entre um par de medidas cuja distância é igual a $h, N(h)$ é o número de pares de medidas cuja distância é igual a $h$, e $\gamma(h)$ é o valor da semivariância correspondente à distância $h$.

Essa função que traduz a semivariância para as várias distâncias entre as observações é designada por variograma experimental ou empírico. Através do variograma experimental observa-se que medidas mais próximas tendem a ser mais semelhantes do que as que estão mais afastadas, e à medida que a distância aumenta, o valor da semivariância tende a aumentar, visto que a diferença entre medidas tende a ser superior. Ao contrário, quando a distância entre as medidas diminui, o valor da semivariância tende a diminuir, uma vez que a diferença entre medidas tende a ser inferior (KITANIDIS, 1997).

\section{Modelos teóricos de Semivariograma}

Uma vez construído o semivariograma experimental, é necessário determinar um modelo matemático que melhor se ajuste ao comportamento da dependência espacial dos dados. Os modelos: esférico (Equação 2), exponencial (Equação 3) e gaussiano (Equação 4), apresentados respectivamente nas equações a seguir, são os modelos matemáticos geralmente utilizados nos ajustes semivariograma experimental pois se adaptam muito bem a dados ambientais:

$$
\begin{gathered}
\gamma(h)=\left\{\begin{array}{c}
C_{0}+C_{1}\left[\frac{3}{2}\left(\frac{\|h\|}{a}\right)-\frac{1}{2}\left(\frac{\|h\|}{a}\right)^{3}\right], 0 \leq h<a \\
C_{0}+C_{1}, h \geq a
\end{array}\right. \text { Equação (2) } \\
\gamma(h)=\left\{C_{0}+C_{1}\left[1-\exp \left(-\frac{3\|h\|}{a}\right)\right], h \geq 0\right. \text { Equação (3) } \\
\gamma(h)=\left\{C_{0}+C_{1}\left[1-\exp \left(-3\left(\frac{\|h\|}{a}\right)^{2}\right)\right], h \geq 0\right. \text { Equação (4) }
\end{gathered}
$$

De acordo com esses modelos são determinados três parâmetros, efeito pepita ou "nugget", $C_{0}$, contribuição ou "sill partial", $C_{1}$, e alcance ou "range", a. Esses parâmetros serão utilizados no cálculo das estimativas por krigagem (KITANIDIS, 1997; ISAAKS et al., 1989; CLARK et al., 2000; YAMAMOTO et al., 2013 ; LISBOA et al., 2016).

\section{Validação dos Modelos Teóricos}

O procedimento de validação é uma etapa importante no processo de análise geoestatística visto que os métodos de interpolação requerem técnicas de confiabilidade que possibilitem comparar o desempenho das estimativas obtidas por krigagem usando os modelos de semivariograma ajustados. A 
validação cruzada é uma técnica que permite esta avaliação e comparação. Nesta técnica, o processo de krigagem é usado para estimar o valor do parâmetro em estudo nos pontos amostrados. Nesse processo, cada uma das observações é temporariamente retirada do conjunto de dados e o seu valor é estimado utilizando as restantes, repetindo-se para todas as observações. Os valores estimados podem ser analisados efetuando uma análise estatística dos erros. Nessa análise estatística dos erros são normalmente calculados os seguintes indicadores estatísticos: erro médio (EM) (Equação 5), raiz quadrada do erro quadrático médio (REQM) (Equação 6) e o erro estandardizado médio (ESM) (Equação 7), dados pelas equações a seguir (WACKERNAGEL, 2003; LUNDGREN et al., 2017):

$$
\begin{gathered}
E M=\frac{1}{N} \sum_{i=1}^{N}\left[\hat{Z}\left(x_{i}\right)-Z\left(x_{i}\right)\right] \text { Equação (5) } \\
R E Q M=\sqrt{\frac{1}{N} \sum_{i=1}^{N}\left[\hat{Z}\left(x_{i}\right)-Z\left(x_{i}\right)\right]^{2}} \text { Equação (6) } \\
E S M=\frac{1}{N} \sum_{i=1}^{N}\left[\frac{\widehat{Z}\left(x_{i}\right)-Z\left(x_{i}\right)}{\sigma^{2}\left(x_{i}\right)}\right] \text { Equação (7) }
\end{gathered}
$$

onde $\hat{Z}\left(x_{i}\right)$ é o valor estimado e $Z\left(x_{i}\right)$ o valor observado na posição $x_{i}$; N é o número de observações estimadas; e $\sigma^{2}\left(x_{i}\right)$ é a variância da estimativa para o ponto $x_{i}$ (ou variância do erro de estimação) definida pela equação 8 seguir:

$$
\sigma^{2}\left(x_{i}\right)=\operatorname{det}\left(X^{t} \mathrm{M}-2 \lambda I_{1}\right)=\sum_{i=1}^{M} \alpha_{i} \gamma\left(x_{i}, x_{0}\right)-\lambda \text { Equação (8) }
$$

O desejável é que EM seja próximo a zero, indicando que o estimador é não enviesado. O valor de REQM deve ser o menor possível, indicando proximidade entre valores estimados e medidos.

\section{Interpolação por Krigagem}

A krigagem é um método de inferência espacial que estima informações em pontos não amostrados a partir de informações situadas em pontos amostradas, considerando a estrutura de dependência espacial do fenômeno, ou seja, com base no resultado da análise variográfica.

O estimador geoestatístico é designado por krigagem. Este estimador pode assumir várias formas sendo a krigagem normal (ou ordinária) a mais habitual (YAMAMOTO et al., 2013). A krigagem normal (ordinária) é um estimador "BLUE" (best linear unbiased estimator). É dito linear, visto que as suas estimativas são combinações lineares ponderadas das medidas. É dito não enviesado pois procura que a média dos erros de estimação seja igual a zero. É dito o melhor porque pretende que a variância dos erros de estimação seja mínima (SOARES, 2000). Assim, o valor da estimativa $\hat{Z}\left(x_{0}\right)$ num ponto não amostrado localizado em ${ }^{\mathrm{x}_{0}}$ é dado por (Equação 9):

$$
\hat{Z}\left(x_{0}\right)=\sum_{i=1}^{M} \propto_{i} Z\left(x_{1}\right) \quad \text { Equação } 9
$$

onde $\mathrm{Z}\left(\mathrm{x}_{\mathrm{i}}\right), \mathrm{i}=1,2, \ldots, \mathrm{M}$ são as observações vizinhas à localização $\mathrm{x}_{0}$ usadas na krigagem $\mathrm{e}$ $\alpha_{i}, i=1,2, \ldots, M$ os respectivos pesos (DOSZA et al., 2016).

\section{RESULTADOS E DISCUSSÃO}

\section{Análise Estatística Descritiva e Espacial}

Inicialmente foi realizada uma análise estatística descritiva para cada um dos metais analisados nos dois cenários. De acordo Paz-Gonzalez et al. (2001) a estimação através do método de krigagem apresenta 
resultados mais satisfatórios quando os dados seguem uma distribuição próxima à distribuição gaussiana. Quando os dados apresentam assimetria, a média e variância dos valores não são muito adequadas para caracterizar a continuidade espacial. Dessa forma, foi realizada a estatística descritiva dos dados para verificar o comportamento da distribuição. A Tabela 1 apresenta um resumo estatístico para os dois cenários.

Tabela 1: Sumário estatístico dos metais analisados em mg. $\mathrm{kg}^{-1}$ para os dois cenários (Araucária e Colombo). CENÁRIO 1 - ARAUCÁRIA

\begin{tabular}{|c|c|c|c|c|c|}
\hline Sumário estatístico & Cr mg.kg ${ }^{-1}$ & Cu mg.kg-1 & Ni mg.kg ${ }^{-1}$ & Pb mg.kg-1 & Zn mg.kg-1 \\
\hline Mínimo & 16,08 & 11,10 & 10,80 & 2,79 & 32,93 \\
\hline Máximo & 294,48 & 135,71 & 76,81 & 227,00 & 311,54 \\
\hline Média & \begin{tabular}{|l|}
99.17 \\
\end{tabular} & 32,60 & 35,47 & 30,78 & \begin{tabular}{|l|}
102,18 \\
\end{tabular} \\
\hline Mediana & 71,27 & 21,39 & 31,48 & 15,62 & 75,04 \\
\hline Coeficiente de Assimetria & 1,29 & 2,03 & 0,70 & 2,90 & 1,49 \\
\hline Curtose & 0,77 & 2,81 & $-0,42$ & 7,09 & 1,02 \\
\hline 1ㅇ quartil & 65,65 & 16,54 & 24,92 & 12,38 & 57,58 \\
\hline 3ㅇ quartil & 116,54 & 24,63 & 45,24 & 22,25 & 89,64 \\
\hline \multicolumn{6}{|l|}{ CENÁRIO 2-COLOMBO } \\
\hline Sumário estatístico & Cr mg.kg-1 & Cu mg.kg-1 & Ni mg.kg-1 & Pb mg.kg-1 & Znmg.kg-1 \\
\hline Mínimo & 3,80 & 8,63 & 3,19 & 1,19 & 6,01 \\
\hline Máximo & 24,36 & 104,19 & 7,96 & 19,91 & 188,96 \\
\hline Média & 12,80 & 18,39 & 5,90 & 9,91 & 54,01 \\
\hline Mediana & 12,07 & 13,89 & 6,17 & 8,96 & 41,95 \\
\hline Coeficiente de Assimetria & 0,46 & 3,6 & $-0,56$ & 0,37 & 1,6 \\
\hline Curtose & $-0,31$ & 11,83 & $-0,38$ & $-0,67$ & 1,9 \\
\hline 10 quartil & 9,18 & 11,66 & 5,14 & 7,36 & 30,08 \\
\hline 3ㅇquartil & 15,64 & 15,74 & 6,69 & 12,82 & 56,99 \\
\hline
\end{tabular}

De acordo com Paz-Gonzalez et al. (2001) a estimação através da krigagem apresenta melhores resultados quando os dados seguem uma distribuição aproximadamente normal. Entretanto, não raramente em ciências ambientais, dados seguem a uma distribuição lognormal. Nessa análise foi investigado o comportamento dos dados quanto a normalidade considerando-se transformações logarítmicas que não apresentaram alterações significativas. Considerou-se ainda transformações mais gerais de dados, como da família Box-Cox, que incluem a transformação logarítmica, mas estas também não impactaram as análises de forma que se optou por utilizar os dados originais não transformados (ZHANG et al., 1998). No conjunto de dados do cenário 1 , os valores da média e mediana são próximos para o Ni. Já no conjunto de dados do cenário 2, os valores da média e mediana são próximos para os cinco metais. Esse resultado indica uma distribuição próxima a normal e desejável para aplicabilidade da metodologia geoestatística. Valores discrepantes no conjunto de medidas podem gerar variâncias nos resultados. Esse caso foi verificado com o $\mathrm{Cr}, \mathrm{Cu}, \mathrm{Pb}$ e $\mathrm{Zn}$ no cenário 1 que apresentaram um valor extremamente alto em uma das suas amostras. Esse valor afetou nos valores da média e mediana do conjunto de dados que não ficaram tão próximos, e, consequentemente afetou a assimetria da distribuição. Os valores dos coeficientes de assimetria e curtose, suficientemente próximos de 0 e 3 , respectivamente, indicam uma distribuição aproximadamente normal dos dados. Podemos observar na Tabela 1 que a existência dos valores discrepantes no conjunto de dados, conforme mencionado para o $\mathrm{Cr}, \mathrm{Cu}, \mathrm{Pb}$ e $\mathrm{Zn}$ no cenário 1, indicam assimetrias nas distribuições dos dados desses elementos (PEDROSA, 2016).

Faz-se necessário compreender melhor o comportamento espacial dos dados para verificar a existência ou não de dependência espacial. Foram construídos gráficos de quartis para os elementos- traço 
analisados nos dois cenários visando identificar a estrutura de correlação espacial, ou seja, a medida estatística, associada à covariância espacial, que melhor descreva os dados, a fim de estimar a magnitude da autocorrelação espacial entre as áreas (LUZARDO et al., 2017).

Continuando a análise exploratória dos dados foram realizados gráficos de quartis para os elementos analisados. Observando os gráficos de quartis da Figura 4 é possível visualizar a localização dos pontos amostrais de maior concentração do elemento $\left(\mathrm{mg} \cdot \mathrm{kg}^{-1}\right)$. Esses valores, na Figura 4, estão representados pelas esferas maiores (3ㅇ e 4 ㅇ quartil).

Na Tabela 1 são apresentados os valores dos quartis para os elementos analisados. Para o $\mathrm{Cr}$ as concentrações superiores do elemento encontram-se localizados na parte inferior da área de coleta, sendo valores a partir de $116,54 \mathrm{mg} \mathrm{kg}^{-1}$; o Cu possui concentrações maiores localizadas no centro e na parte inferior da área de coleta com 24,63 mg. $\mathrm{kg}^{-1}$; o Ni possui os maiores valores de concentração acima de 45,24 mg. $\mathrm{kg}^{-}$ ${ }^{1}$ localizados no centro e na parte inferior da área de coleta; já o $\mathrm{Pb}$ possui os maiores valores de concentração acima de 22,25 mg. $\mathrm{kg}^{-1}$ localizados na região central e na parte inferior da área de coleta; e o Zn possui os maiores valores de concentração acima de $89,64 \mathrm{mg} \cdot \mathrm{kg}^{-1}$ posicionados, também no centro e na parte inferior da área. Esses pontos próximos com valores similares dão-nos evidências de que existe dependência espacial.

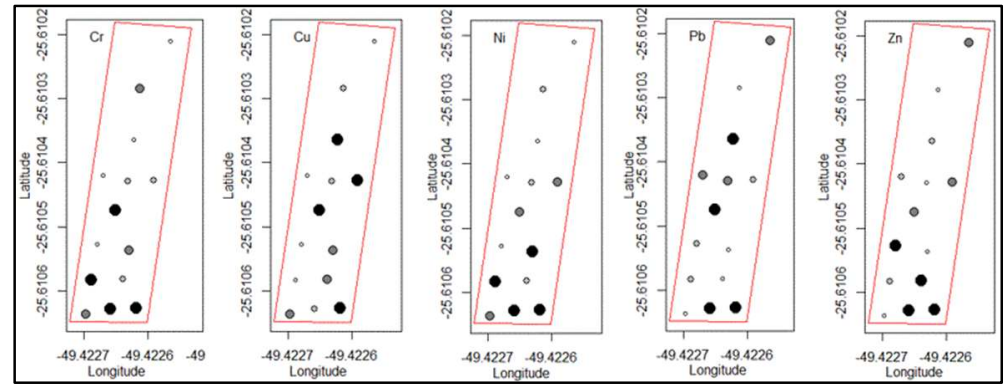

Figura 4: Gráficos de dispersão dos quartis da amostragem referente ao cenário 1 - AR.

Na Figura 5 pode ser visualizado os gráficos de dispersão dos quartis da amostragem referente ao cenário 2. Os pontos amostrais de maior concentração estão representados pelas esferas maiores (3으 e 4응 quartil) em mg. $\mathrm{kg}^{-1}$. Essa visualização nos possibilita verificar a existência de dependência espacial no conjunto de dados.

$\mathrm{Na}$ Tabela 1 são apresentados os valores dos quartis para os elementos analisados. O elemento $\mathrm{Cr}$ possui as concentrações superiores localizadas na parte superior e lateral direita da área de coleta, sendo valores a partir de 15,64 mg. $\mathrm{kg}^{-1}$; o Cu possui as concentrações maiores localizadas na parte superior da área com pois pontos de alta concentração na área lateral da amostragem sendo os valores a partir de 15,74 $\mathrm{mg} . \mathrm{kg}^{-1}$; as concentrações maiores do $\mathrm{Ni}$ com valores a partir 6,69 mg. $\mathrm{kg}^{-1}$ localizam-se a partir da metade da parte superior seguindo a parte lateral da área até a metade da área inferior; já o $\mathrm{Pb}$ possui os maiores valores de concentração acima de $12,82 \mathrm{mg} \cdot \mathrm{kg}^{-1}$, localizados na parte superior da área com 01 ponto de maior concentração isolado, na parte inferior da área amostral de coleta; e o $\mathrm{Zn}$ possui os maiores valores de concentração acima de 56,99 mg. $\mathrm{kg}^{-1}$ posicionados na parte superior até a metade da parte lateral da área da figura. $O$ cenário 2 possui a distribuição das amostras onde pontos próximos possuem valores similares 
evidenciando, novamente, a existência de dependência espacial.

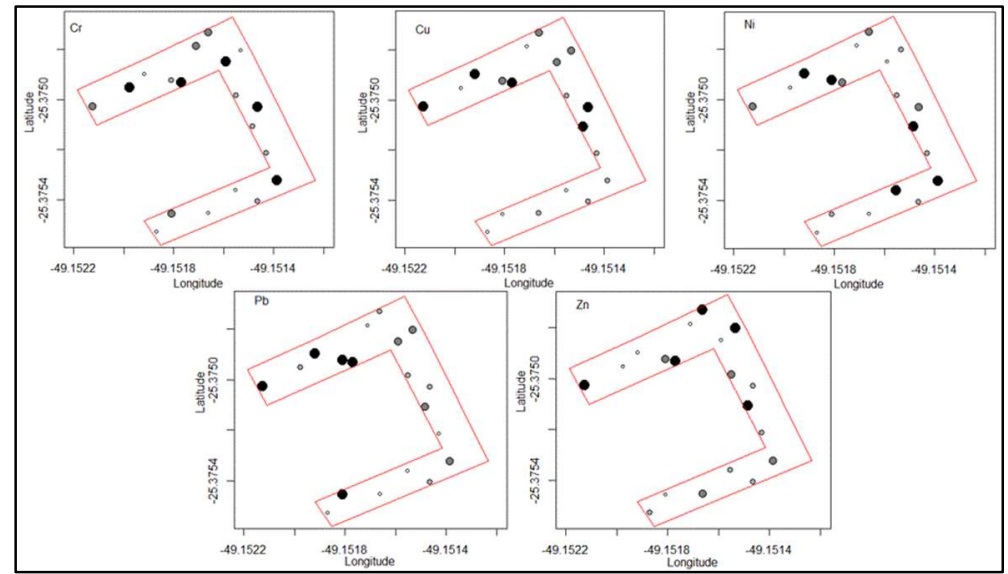

Figura 5: Gráficos de dispersão dos quartis da amostragem referente ao cenário 2 - CL.

\section{Análise e Modelagem do Semivariograma Experimental}

Após a verificação da existência de dependência espacial no conjunto de dados, o passo seguinte foi quantificar essa dependência espacial através da construção dos semivariogramas experimentais. Foram gerados os semivariogramas experimentais para os dados, dos dois cenários, e adotou-se o modelo isotrópico após verificar que o fenômeno apresentava um comportamento semelhante em todas as direções. Estabeleceu-se o ponto de corte dos semivariogramas para os três modelos testados, esférico, exponencial e gaussiano, através de uma análise visual levando-se em consideração uma fração da máxima distância e a sua estabilização após certa distância (SOARES, 2000). Os modelos matemáticos foram ajustados através do método dos mínimos quadrados. Foi realizado o procedimento de validação cruzada através dos parâmetros calculados pelas equações apresentadas no item $\mathbf{d}$. Realizada a análise dos indicadores estatísticos dos erros (EM, REQM e ESM) foi possível escolher o melhor modelo dentre os testados.

Na Figura 6 apresentam-se os semivariogramas experimentais e os modelos ajustados para os elementos-traço analisados no cenário1.

Observando os semivariogramas modelados para os modelos testados, o $C_{0}$ (efeito pepita) encontrado foi igual a zero, mostrando que a variabilidade a pequenas distâncias é nula. Para a construção do semivariograma do $\mathrm{Pb}$ foi retirado 01 ponto do conjunto amostral, cujo valor desse elemento nesse ponto era de $227,00 \mathrm{mg} \cdot \mathrm{kg}^{-1}$, muito discrepante em relação aos demais. Após a retirada do ponto, o valor máximo da concentração passou de $227,00 \mathrm{mg} \cdot \mathrm{kg}^{-1}$ para $29,09 \mathrm{mg} \cdot \mathrm{kg}^{-1}$. A retirada do ponto do conjunto amostral foi importante pois esse valor atípico encontrado pode ser resultante de um vazamento de uma das baterias dos carros apreendidos e estocados no pátio, uma vez que as baterias automotivas são normalmente do tipo chumbo-ácido, o que faz com que sejam classificadas como de elevado risco ambiental (FERNANDES et al., 2011). Dessa forma, optou-se pela retirada deste ponto, já que ele não representava o conjunto de dados. Nos semivariogramas da Figura 6 observa-se que o elemento $\mathrm{Ni}$ apresentou uma variabilidade bem menos acentuada e o $\mathrm{Zn}$ a variabilidade mais alta. Os valores do alcance dos semivariogramas para os elementos $\mathrm{Cr}$, $\mathrm{Ni}$ e $\mathrm{Zn}$ foram em média $12 \mathrm{~m}$, ao passo que para o $\mathrm{Cu}$ e $\mathrm{Pb}$ o alcance foi em média de $22 \mathrm{~m}$. O parâmetro 
alcance no semivariograma representa a distância dentro da qual as amostras se apresentam correlacionadas espacialmente. A análise variográfica mostra correlações espaciais semelhantes entre o Cr, Ni e entre o Cu e $\mathrm{Pb}$.

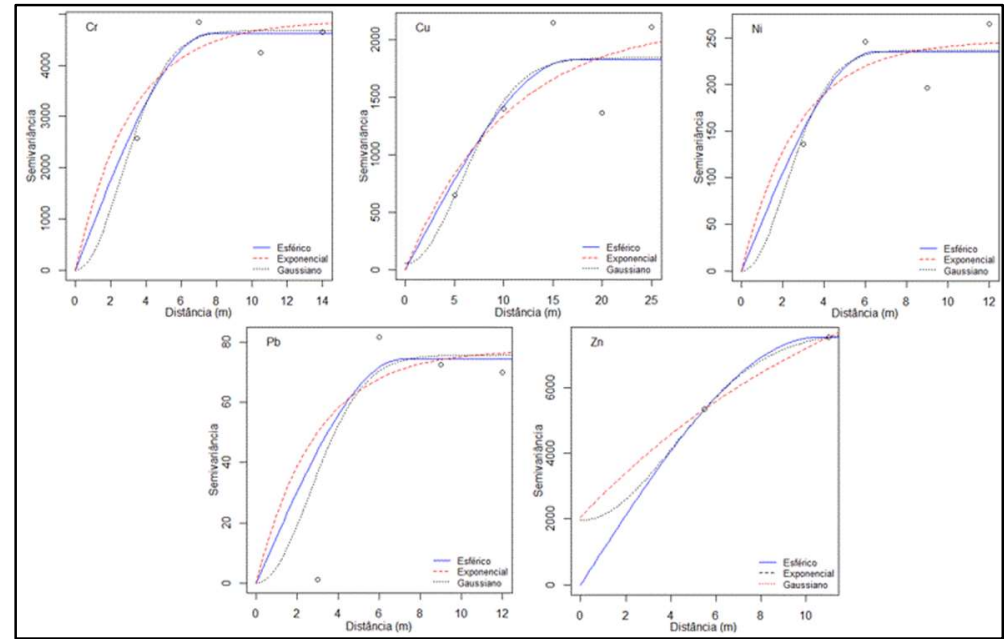

Figura 6: Semivariogramas e modelos ajustados para os elementos-traço analisados no cenário 1.

Na Figura 7 pode ser visualizado os semivariogramas experimentais e os modelos ajustados para os elementos-traço analisados no cenário 2. O elemento $\mathrm{Ni}$ apresentou uma pequena variabilidade e o Zn a variabilidade mais alta. Isto também foi observado para esses dois elementos na análise do cenário 1. Os valores do alcance dos semivariogramas para os elementos $\mathrm{Pb}$ e Zn foram em média $22 \mathrm{~m}$, para o Cr o alcance foi em torno de $30 \mathrm{~m}$, o Cu e o Ni tiveram alcances maiores, em torno de $60 \mathrm{~m}$.

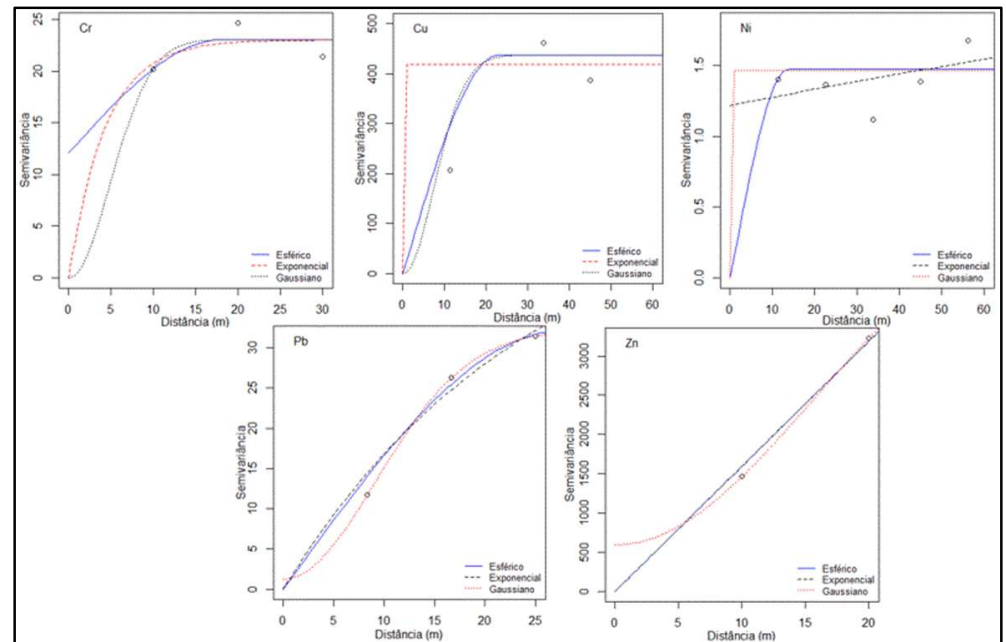

Figura 7: Semivariogramas e modelos ajustados para os elementos-traço analisados no cenário 2. Krigagem: Mapeamento da distribuição dos parâmetros

Realizada a estatística dos erros através do processo de validação cruzada foi possível a escolha do modelo que melhor se adequasse ao conjunto de dados. Ela já foi validada em diversos estudos geoestatísticos. A técnica de validação cruzada para determinar a precisão das estimativas do erro de krigagem também foi testada por Lundgren et al. (2017) com sucesso.

Para todas as análises o modelo esférico e exponencial, apresentaram um bom resultado no cálculo das estimativas. Isaaks et al. (1989) já apontavam os modelos esférico e exponencial como adequados na modelagem de fenômenos ambientais. Mello et al. (2019) relatam que o modelo esférico e exponencial se 
apresentara adequados na modelagem apresentando superfícies interpoladas mais suavizadas. O modelo esférico também se apresentou adequado na modelagem da distribuição espacial de dois metais pesados (cobre e cádmio) ao longo das margens de um rio (MANUEL et al., 2017).

O próximo passo, foi gerar os mapas através da krigagem, e utilizou-se os parâmetros do modelo de semivariograma esférico, para estimar a distribuição da dispersão dos elementos-traço nas amostras de solo, conforme Figura 8. Através da visualização dos mapas gerados observa-se claramente a variação espacial da concentração dos elementos.

Os mapas mostram os locais com maiores valores de concentração dando indícios de possíveis áreas de risco à saúde pública. De acordo com Lourenço et al. (2005) esses mapas dão uma leitura prévia de regiões com altos índices do contaminante, sendo muito úteis em estudos ambientais. Observando os mapas da Figura 8 e comparando o sumário estatístico da Tabela 1, pode-se perceber que a amplitude das estimativas se encontra na faixa da amplitude da amostragem. Isso revela que a análise geoestatística forneceu estimativas confiáveis da dispersão desses poluentes.

Importante destacar que as regiões mais escuras nos mapas (Figuras 8 e 9), são as que tiveram maior teor dos elementos, porém não necessariamente atingiram os valores acima do que a legislação ambiental permite. O Valor de Investigação (VI) é a concentração de uma determinada substância no solo que, em níveis elevados, apresenta riscos potenciais à saúde humana. O Valor de Prevenção (VP) é o valor limite de uma determinada substância, de modo que seja capaz de sustentar as suas funções principais e foi estabelecido baseado em análise de fitotoxicidade e avaliação de risco ecológico.

Fazendo-se o comparativo dos teores dos elementos (em mg. $\mathrm{kg}^{-1}$ ) obtidos no cenário 1 (Araucária) com os previstos na Resolução CONAMA 420/2009, a região de AR apresentou concentração dos elementos analisados entre os valores de prevenção (VP) e investigação (VI), com destaque para os metais $\mathrm{Ni}$ e $\mathrm{Cr}$, onde em alguns pontos, ambos ultrapassaram os $\mathrm{VI}$. Para os metais $\mathrm{Cu}, \mathrm{Pb}$ e $\mathrm{Zn}$, os valores chegaram a ultrapassar o VP em alguns pontos. A região de AR classifica-se na classe 4 conforme a Resolução CONAMA 420 (2009).

Para a geração dos mapas das estimativas da distribuição dos teores dos elementos-traço para a coleta do cenário 2 , o procedimento foi o mesmo do cenário 1. Primeiramente, foi realizada a estatística dos erros através do processo de validação cruzada para a escolha do melhor modelo. Novamente os modelos esférico e exponencial apresentaram um bom resultado no cálculo das estimativas. Optou-se pelos parâmetros do modelo de semivariograma esférico para a geração dos mapas, conforme Figura 9. Ao visualizar os mapas gerados observa-se a variação espacial da concentração dos elementos. Comparando os mapas da Figura 9 com o sumário estatístico da Tabela 1 percebeu-se que a amplitude das estimativas se encontra na faixa da amplitude da amostragem. A análise geoestatística do conjunto de dados do cenário 2 , novamente forneceu estimativas confiáveis da dispersão desses poluentes, com valores estimados de amplitude próxima aos coletados.

Fazendo-se o mesmo comparativo do Cenário 2 (Colombo) com a legislação ambiental vigente no Brasil, Resolução CONAMA 420/2009, a região de CL manteve-se com a concentração dos metais analisados abaixo do VP, com exceção para o elemento $\mathrm{Cu}$, sendo classificada na classe 3 , devido o teor do elemento $\mathrm{Cu}$ 
apresentar valor superior ao VP e os demais elementos ( $\mathrm{Ni}, \mathrm{Cr}$, Zn e Pb) apresentaram valores abaixo do VP. Importante destacar que quando se observa o mapa de estimativas (Figura 9) para o elemento $\mathrm{Cu}$, a área mais escura do mapa está localizada em apenas um ponto, devido à escala de valores utilizada. Desta forma, ressalta-se a necessidade de sempre confrontar o mapa geoestatístico com a legislação vigente, já que para cada elemento químico, os valores tabelados são diferentes.

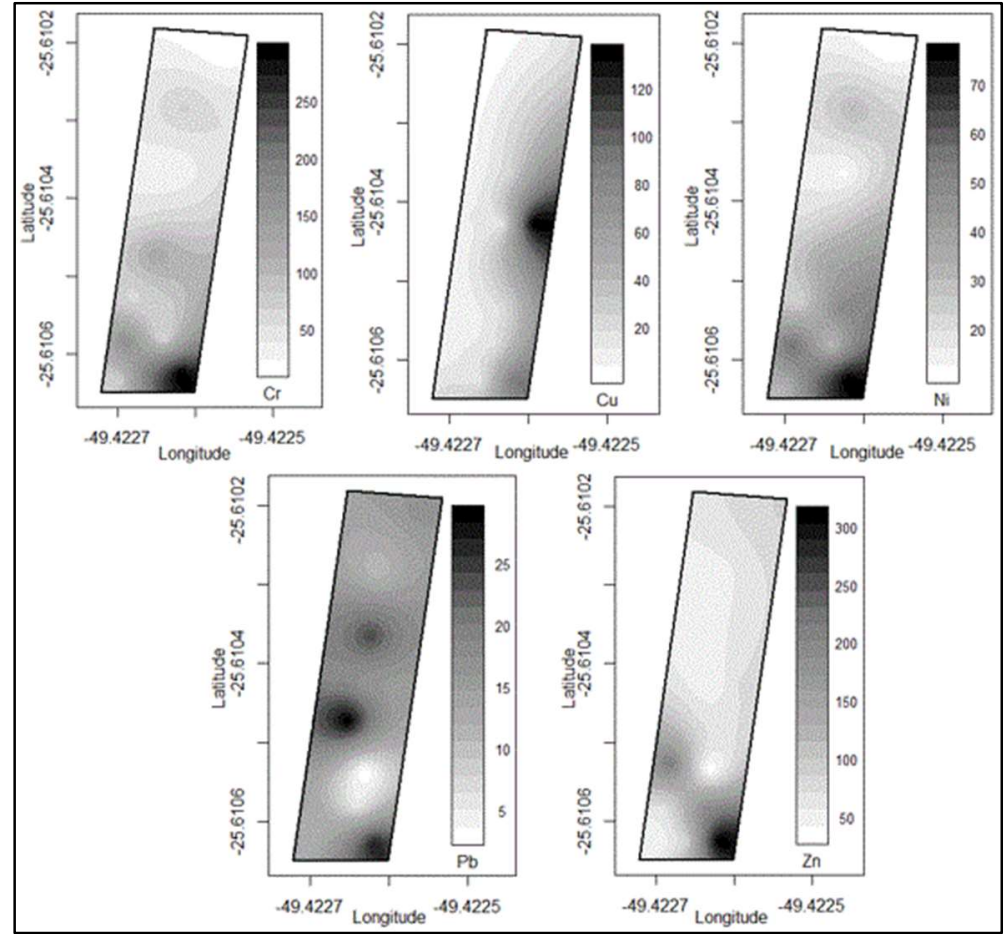

Figura 8: Mapas das estimativas da distribuição dos teores dos elementos-traço para a coleta do cenário 1 utilizando a krigagem ordinária e o modelo esférico.

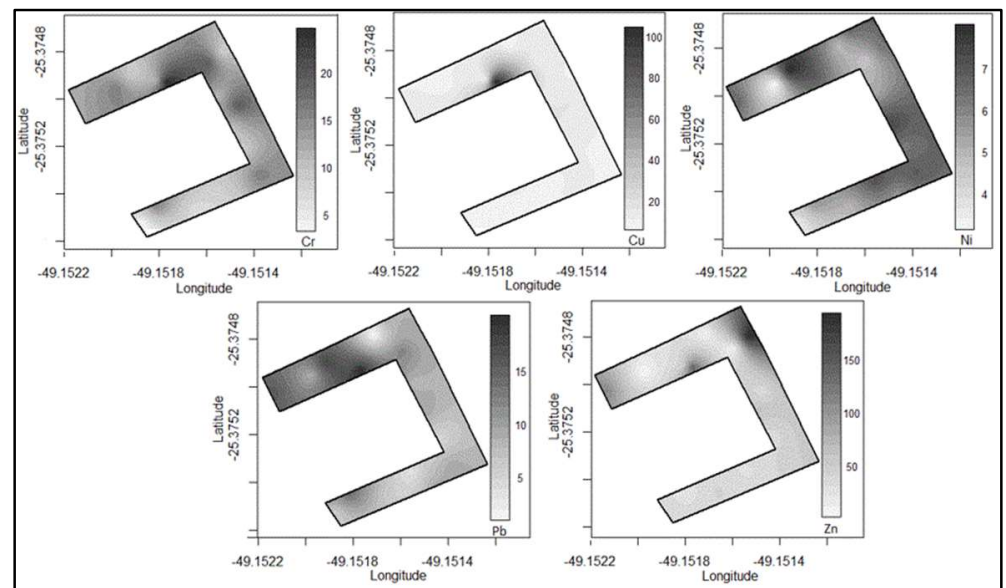

Figura 9. Mapas das estimativas da distribuição dos teores dos elementos- traço para a coleta do cenário 2 utilizando a krigagem ordinária e o modelo esférico.

Após a classificação dos solos conforme Art. 20 disposto na Resolução CONAMA 420 (2009), procedimentos de prevenção e controle da qualidade do solo devem ser adotados. No caso do local CL, este requer identificação da fonte potencial de contaminação, avaliação da ocorrência natural da substância, controle das fontes de contaminação e monitoramento da qualidade do solo e da água subterrânea (MAKARA et al., 2020). 


\section{CONCLUSÕES}

A análise geoestatística dos elementos-traço revelou que modelo de semivariograma esférico apresentou o menor erro das estimativas no processo de validação cruzada. Foi possível verificar a dependência espacial entre as observações amostrais, quantificar essa dependência através dos semivariogramas e gerar mapas da distribuição espacial por krigagem ordinária o qual representam, com confiabilidade, a distribuição da concentração dos respectivos poluentes para os dois cenários. Os resultados obtidos foram muito satisfatórios e a metodologia geoestatística mostrou-se eficiente neste estudo.

Esses resultados nos sugerem que a metodologia geoestatística, traz contribuições relevantes com informações muito precisas e pode ser utilizada para estudos que envolvam a área de controle e monitoramento me elementos-traço em solos urbanos. Entretanto, é importante cruzar os mapas obtidos com a legislação vigente quanto aos valores orientadores de elementos químicos no solo para poder guiar políticas públicas quanto ao monitoramento de áreas contaminadas, uma vez que a legislação classifica os solos de acordo com o grau de contaminação em diferentes categorias e orienta sobre ações a serem tomadas conforme a quantidade de cada metal.

AGRADECIMENTOS: Os autores agradecem ao Laboratório de Solos (LASOL) da Universidade Tecnológica Federal do Paraná (UTFPR).

\section{REFERÊNCIAS}

BATISTA, P. H. D.; MONTENEGRO, A. A. A.; ALMEIDA, G. L. P.; TAVARES, U. E.; RODRIGUES, R. A. S.. Variabilidade espacial da resistência à penetração e teor de água em neossolo cultivado com banana no Agreste Pernambucano. Revista Engenharia na Agricultura-Reveng, v.27, n.1, p.54-60, 2019. DOI: http://doi.org/10.13083/reveng.v27i1.881

BUSSAB, W. O.; MORETTIN, P. A.. Estatística Básica. 9 ed. São Paulo: Saraiva, 2017.

CLARK, I.; HARPER, W.. Practical Geostatiscs 2000. Ecosse North America Llc, 2000.

CONAMA. Conselho Nacional de Meio Ambiente. Resolução Conama 420. CONAMA, 2009.

DOZSA, B.; MONEGO, M. L. C.; KUMMER, L.. Modelagem geoestatística da ocorrência de casos de leptospirose e alagamentos no município de Curitiba - Paraná, no ano de 2014. Revista Holos, v.1, p.381-393, 2016. DOI:

https://doi.org/10.15628/holos.2016.3857

FERNÁNDES, Z. H.. Análise de metais pesados em solos de Pernambuco com diferentes atividades antrópicas. Tese (Doutorado em Tecnologias Energéticas e Nucleares) Universidade Federal De Pernambuco, Recife, 2017.

FERNANDES, J. D.; DANTAS, E. R. B.; BARBOSA, J. N.; BARBOSA, E. A.. Estudo de impactos ambientais em solos: o caso da reciclagem de baterias automotivas usadas, tipo chumbo-ácido. Revista Brasileira de Gestão e

Desenvolvimento Regional, v.7, n.1, p.231-255, 2011.
ISAAKS, E. H.; SRIVASTAVA, R. M.. Applied Geostatistics. London: Oxford University Press, 1989.

KITANIDIS, P.. Introduction to geostatistics: Applications in hydrogeology. New York: Cambridge University Press, 1997.

LADO, L. R.; HENGL, T.; REUTER, H. I.. Heavy metals in European soils: A geostatistical analysis of the FOREGS Geochemical database. Geoderma, v.148, p.189-199, 2008. DOI: http://doi.org/10.1016/j.geoderma.2008.09.020

LIN, Y.; CHANG, T.; SHIH, C.; TSENG, C.. Factorial and indicator kriging methods using a geographic information system to delineate spatial variation and pollution sources of soil heavy metals. Environmental Geology, v.42, n.8, p.900909, 2002. DOI: http://doi.org/10.1007/s00254-002-0600-5

LISBOA, E. G; CARVALHO, J. M. C. M.; MENDES, R. L. R.. O Uso da Geoestatística na Avaliação dos Parâmetros Hidrogeológicos para Compor o Mapa de Vulnerabilidade Intrínseca de Aquíferos. RBRH, v.21, n.1, p.188-199, 2016. DOI: http://dx.doi.org/10.21168/rbrh.v21n1.p188-199

LOURENÇO, R. W.; LANDIM, P. M. B.. Mapeamento de áreas de risco à saúde pública por meio de métodos geoestatísticos. Cadernos de Saúde Pública, v.21, n.1, p.150160, 2005. DOI: http://doi.org/10.1590/S0102$\underline{311 \times 2005000100017}$

LUNDGREN, W. J. C.; SILVA, J. A. A.; FERREIRA, R. L. C.. A Precisão da Estimativa do Erro da Krigagem pela Validação 
Cruzada. Revista Floresta e Ambiente, v.24, 2017. DOI: http://dx.doi.org/10.1590/2179-8087.124114

LUZARDO, A. J. R.; CASTAÑEDA FILHO, R. M.; RUBIM, I. B.. Análise espacial exploratória com o emprego do Índice de Moran. Revista GEOgraphia, v.19, n.40, 2017. DOI: http://doi.org/10.22409/GEOgraphia2017.1940.a13807

MAKARA, C. N.; DEL MONEGO, M. L. C.; KUMMER, L.. Estudo do risco ambiental atrelado ao armazenamento de veículos sobre o solo. Revista Ibero-Americana de Ciências Ambientais, v.11, n.05, 2020.

MANUEL, L.; SCALON, J. D.; GONZALEZ, G. G. H.; MOREIRA, J. M.; RIBEIRO, M. C.; MELO, A. F.. Estudo da variabilidade espacial da concentração de cobre e cádmio ao longo da margem do rio Meuse. PUBVET, v.11, n.08, p.744-839, 2017. DOI: http://dx.doi.org/10.22256/PUBVET.V11N8.802-807

MELLO, Y. R.; SAMPAIO, T. V. M.. Análise geoestatística da precipitação média para o estado do Paraná. Revista Brasileira de Climatologia, v.25, 2019. DOI: http://dx.doi.org/10.5380/abclima.v25i0.64468

MILHOME, M. A. L.; HOLANDA, J. W. B.; ARAÚJO NETO, J. R.; NASCIMENTO, R. F.. Diagnóstico da Contaminação do Solo por Metais Tóxicos Provenientes de Resíduos Sólidos Urbanos e a Influência da Matéria Orgânica. Rev. Virtual Quim., v.10, n.1, p.59-72, 2018. DOI: http://doi.org/10.21577/1984-6835.20180007

MONEGO, M. L. C. D.; RAMOS, P.; NEVES, M. V.. Avaliação de impacto ambiental de descargas de águas residuais usando uma metodologia geoestatística. Revista Brasileira de Recursos Hídricos, v.15, n.3, p.57-68, 2010. DOI: http://dx.doi.org/10.21168/rbrh.v15n3.p57-68

PEDROSA, A. C.; GAMA, S. M. A.. Introdução computacional à probabilidade e estatística. Porto, 2016.

PEREIRA, A. C. C.; LIMA, E. S. A.; SANTOS, A. M.; AMARAL SOBRINHO, N. M. B.. Análise e monitoramento de metais pesados no solo. In: COSTA, A. F. S.; COSTA, A. N.. Valores orientadores de qualidade de solos no Espírito Santo. Incaper - Instituto Capixaba de Pesquisa, Assistência Técnica e Extensão Rural, 2015.

R DEVELOPMENT CORE TEAM. R: A language and environment for statistical computing. Vienna: R Foundation for Statistical Computing, 2008.

PAZ-GONZALEZ, A.; CASTRO, M. T. T.; VIEIRA, S. R. Geostatistical analysis of heavy metals in a one-hectare plot under natural vegetation in a serpentine area. Canadian Journal of Soil Science, v.81, n.4, p.469-479, 2001. DOI: http://doi.org/10.4141/S00-095

SOARES, A.. Geoestatística para as ciências da terra e do ambiente. Lisboa: IST Press, 2000.

SOUZA, Z. M.; SOUZA, G.; MARQUES JUNIOR, J.; PEREIRA, G. T.. Número de amostras na análise geoestatística e na krigagem de mapas de atributos do solo. Ciência Rural, v.44, n.2, p.261-268, 2014. DOI: http://doi.org/10.1590/S010384782014000200011

SOUZA, A. K. R.; MORASSUTI, C. Y.; DEUS, W. B.. Poluição do ambiente por metais pesados e utilização de vegetais como bioindicadore. Acta Biomedica Brasiliensia, v.9, n.3, 2018. DOI: http://doi.org/10.18571/acbm.189

YAMAMOTO, J. K.; LANDIM, P. M. B.. Geoestatística: conceitos + aplicações. São Paulo: Oficina de textos, 2013.

ZHANG, C.; SELINUS, O.; SCHEDIN, J.. Statistical analyses for heavy metal contents in till and root samples in an area of southeastern Sweden. Science of The Total Environment, v.212, n.2-3, p.217-232, 1998. DOI: http://doi.org/10.1016/S0048-9697(97)00341-0

WACKERNAGEL, H.. Multivariate geostatistics: An introduction with applications. Berlin: Springer, 2003.

A CBPC - Companhia Brasileira de Produção Científica (CNPJ: 11.221.422/0001-03) detém os direitos materiais desta publicação. Os direitos referem-se à publicação do trabalho em qualquer parte do mundo, incluindo os direitos às renovações, expansões e disseminações da contribuição, bem como outros direitos subsidiários. Todos os trabalhos publicados eletronicamente poderão posteriormente ser publicados em coletâneas impressas sob coordenação da Sustenere Publishing, da Companhia Brasileira de Produção Científica e seus parceiros autorizados. Os (as) autores (as) preservam os direitos autorais, mas não têm permissão para a publicação da contribuição em outro meio, impresso ou digital, em português ou em tradução. 